Research Square

\title{
Clinical Characteristics and Metabolomics Investigation for Patients with Dengue Virus 2 Infection
}

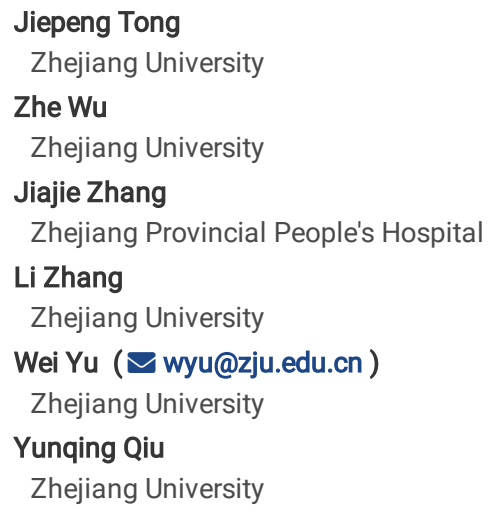




\section{Abstract \\ OBJECTIVES}

Dengue is an endemic viral disease affecting tropical and subtropical regions around the world. The aim of this study was to retrospectively study the clinical characteristics of dengue fever (DF) and evaluate the metabolomic changes.

\section{METHODS}

The medical records of 50 patients presented with dengue virus 2 (DENV-2) RNA positive were reviewed. Serum was collected from 20 patients diagnosed with DF and 20 healthy volunteers. The changes in serum metabolites were explored by UPLC-Q-TOF MS spectrometer.

\section{RESULTS}

Fever, nausea and vomiting, asthenia, skin hyperemia were the most common clinical symptoms. The results of the laboratory examinations showed that leukopenia (76\%, 38/50), thrombocytopenia (92\%, 46/50), increase of aspartate aminotransferase $(96 \%, 48 / 50)$, increase of free fatty acids (FFA) (68\%, $34 / 50)$, increase of creatine kinase (CK) $(44 \%, 22 / 50)$ were more common. Based on orthogonal projection to latent structures-discriminant analysis (OPLSDA), 2 up-regulated and 15 down-regulated metabolites were identified, contributing to DF progress to some extent. Among them, LysoPC (18:2(9Z,12Z)) were highly positive correlated with PE (21:0/20:5(5Z,8Z,11Z,14Z,17Z)) and 13'-Hydroxy-alpha-tocopherol. The identified biomarkers were mainly involved in glycerophospholipids metabolism pathway.

\section{CONCLUSIONS}

The metabolic abnormalities of glycerophospholipids involved in the occurrence of DF caused by DENV-2.

\section{Introduction}

Dengue is one of the important mosquito-borne viral diseases, posing a global health threat to public health. The accurate data on prevalence rate is difficult to obtain due to a vast majority of asymptomatic and self-managed patients. It is estimated that there are 390 million dengue virus (DENV) infections (60 million symptomatic infections) per year in 130 countries [1-2]. Most notably, the mortality is increased from 960 to more than 4032 during $2000-2015$ [3]. In addition, economic burden of dengue is substantial. A systematic analysis showed the annual global cost of dengue was \$8.9 billion (95\% UI, 3.7 billion-19.7 billion) in 2013 [4]. In China, high risk areas of dengue outbreaks were Guangzhou and Yunnan Provinces [5]. However, three outbreaks, especially large epidemic in 2017, were reported in Zhejiang Province with a typical subtropical climate [6].

Dengue virus (DENV) with 4 serotypes (DENV-1, DENV-2, DENV-3, DENV-4) could cause a wide spectrum of disease, including dengue fever (DF), dengue hemorrhagic fever (DHF) and dengue shock syndrome (DSS) [7]. Delayed diagnosis of dengue could increase the risk of severe dengue. Recently, virological tests and serological tests are used for diagnosis of DENV infections in clinic. However, these methods vary in their sensitivity and specificity at different stages [8]. Therefore, it is urgent to develop a rapid and accurate tool for detection of DENV infections in the early stage. The aim of present study was to analyze the clinical characteristics of DF retrospectively and identify metabolites of serum in order to provide rapid diagnosis of DF.

\section{Methods}

\subsection{Medical Records}

The medical records of 50 hospitalized patients presented with DENV-2 RNA positive were reviewed during the outbreak attacked Hangzhou, China in 2017. The data of demographics, clinical characteristics, laboratory results, treatment history, hospitalization and clinical outcomes were collected.

\subsection{Sample preparation and UPLC-Q-TOF MS spectrometer test}

Serum samples were collected from 20 hospitalized DF patients (DF group) and 20 healthy volunteers (HC group) at The First Affiliated Hospital, College of Medicine, Zhejiang University from August to November in 2017. Serum samples were pretreated with triple volumes of acetonitrile and centrifuged at 15,000 rpm for 15 min to extract supernatant. The samples were tested by UPLC-Q-TOF MS spectrometer (Waters, Manchester, UK) using ACQUITY UPLC HSS T3 C18 column $(100 \mathrm{~mm} \times 2.1 \mathrm{~mm}, 1.8 \mu \mathrm{m})$ (Waters, Manchester, UK).

\subsection{Data processing and multivariate analysis}

Nuclear Magnetic Resonance (NMR) data were recorded on AV-400 spectrometer and proton coupling patterns were subjected to multivariate analysis. Metabolites quantification were identified using Chroma TOF (V 4.3x, LECO, United States) for deconvolution and peak matching. Further sorting and annotation were interpreted with Human Metabolome Database (HMDB) (http://www.hmdb.ca/) and LIPID MAPS (http://www.lipidmaps.org/). Differential metabolites responsible for distinguishing between DF patients and healthy volunteers were analysis by principal component analysis (PCA), partial least squares discrimination analysis (PLS-DA), and orthogonal projection to latent structures-discriminant analysis (OPLS-DA) using metaX (http://metax.genomics.cn). R2Y was used to estimate the goodness of fit, and Q2Y was used to assess the quality of prediction [9]. In addition, the 
value of variable importance in the projection (VIP) greater than 1 and log2FC greater than 2 or lower than 0.5 was considered as significant contribution to classification. Furthermore, the potential biomarkers involved in metabolic pathways was performed based on Metabolomics Pathway Analysis (MetPA) (https://www.metaboanalyst.ca/). A p value $<0.05$ was considered statistically significant.

\section{Results}

\subsection{Clinical characteristics of DF}

The clinical characteristics of the enrolled 50 patients showed in Table 1. There were 21 males and 29 females. The average admission days was 4.9 (3.9-7) days. Fever, nausea and vomiting, asthenia, skin hyperemia were the most common clinical symptoms. Regarding the comorbidities, hypertension (26\%, $13 / 50)$, fatty liver $(14 \%, 7 / 50)$, diabetes mellitus $(10 \%, 5 / 50)$ were the most prevalent comorbidities. The results of the laboratory examinations showed that leukopenia $(76 \%, 38 / 50)$, thrombocytopenia (92\%, 46/50), increase of transaminase (alanine aminotransferase 29/50, aspartate aminotransferase 48/50, $\gamma^{-}$ glutamyl transpeptadase $23 / 50)$, increase of free fatty acids (FFA) $(68 \%, 34 / 50)$, increase of creatine kinase (CK) $(44 \%, 22 / 50)$ were more common. The management for most symptomatic DF patients were supportive treatments including.

\subsection{Quality Control (QC) analysis and metabolites annotation}

There was no statistical difference in age and gender between DF group and HC group. The QC samples were used to evaluate the repeatability and stability of UPLC-Q-TOF MS spectrometer. The Pearson correlation coefficient was calculated based on the peak area. The results of QC analysis indicated the experiment conditions exhibited high reliability (Supplementary Figure 1).

A total of 20 classes of metabolites were annotated and 631 types were lipids and lipid-like molecules (Figure 1a). According to the LIPID MAPS, flavonoids, glycerophosphoinositols and glycerophosphocholines were three most metabolites (Figure 1b).

\subsection{Multivariate analysis}

PCA was initially applied to evaluate the distribution pattern among all samples. As shown in Figure 2a, DF group and HC group showed good aggregation and classification. PLS-DA was further carried out to investigate the discrimination between the two groups (Figure 2b). The model parameters for the classification were R2Y $=0.87, \mathrm{Q} 2 \mathrm{Y}=0.79$ by 7-fold cross-validation. There was no obvious overfitting in model validation by randomly shuffled 200 times of the class membership (Figure 2c). The well fit PLS-DA models showed a significant separation in the scores plot between DF patients and HC, indicating the endogenous metabolites were changed as a result of DENV infection. Base on VIP and log2FC, a total of 619 metabolites significantly up-regulated and 64 metabolites down-regulated (Figure 2d).

\subsection{Identification of potential biomarkers}

Based on OPLS-DA, 17 metabolites in the upper-right quadrant of S-plot were tentatively identified, with 2 up-regulated and 15 down-regulated (Figure 2e) (Table 2). The relationship of 17 potential metabolites was evaluated by correlation analysis. Among them, LysoPC (18:2(9Z,12Z)) were highly positive correlated with PE (21:0/20:5(5Z,8Z,11Z,14Z,17Z)) and 13'-Hydroxy-alpha-tocopherol. Further metabolic pathways were analyzed by MetPA. The results from pathway analysis were presented in Figure 2e. Glycerophospholipid metabolism pathway was the main pathway of metabolic disorder in DF.

\section{Discussion}

The global burden of dengue has increased $400 \%$ during 13 years, presenting a great challenge to prevention and control [10]. The current diagnostic procedures for DENV infections had different their own limitations [8]. The results of our retrospective study indicated that the clinical symptoms were mild-tomoderate for most hospitalized patients during the outbreak in Hangzhou. Some degree of leucopenia and thrombocytopenia, increase of transaminase, FFA and CK were common during the febrile phase. In addition, the changes in serum metabolites were investigated by UPLC-Q-TOF MS spectrometer. There were 17 potential biomarkers were identified, mainly involved in glycerophospholipid metabolism pathway.

In the present study, all enrolled patients were infected by DENV-2. Previous study also demonstrated DENV-2 was the dominant serotype in Zhejiang Province, however, other three serotypes were identified during the same period [11]. Most people infected by DENV remain asymptomatic or self-limited [10]. We found fever, nausea and vomiting, asthenia, skin hyperemia were the most common clinical symptoms in hospitalized patients. These symptoms were relieved after supportive treatments. However, critical phase of dengue could be developed from febrile, especially for infants, old age, pregnant women with hypertension or vascular disorders.[10]. Although there were $26 \%$ patients with hypertension in our study, none of hospitalized patients progressed to severe dengue.

Significantly lower white blood cell and platelet were observed in present and previous studies [12]. In addition, laboratory investigations showed transaminase increased, especially for aspartate aminotransferase, indicating the combination of liver and musculoskeletal involvement [13].

Previous metabolomic studies detected serum metabolome and lipidome changes in patients infected with DENV [14]. The changes in host lipid homeostasis could not only satisfy the replication of offspring virus, but also support the energy of lipolysis supply [15-16]. In present study, 2 up-regulated and 15 downregulated serum metabolites were contributed to DF. The down-regulated serum metabolites were phosphatidylcholine (PC), phosphatidylethanolamine (PE), sphingomyelin (SM), lysophospholipids (LPL), which might be attributable to the alternation of cell membrane composition caused by DENV, consistent with previous results in human serum [14]. In addition, glycerophospholipid metabolism pathway was the main pathway of metabolic disorder in DF. Furthermore, the potential glycerophospholipids metabolites have been identified as useful biomarkers for detection of DHF from DF and HC [17]. The glycerophospholipids 
were linked to thrombocytopenia, vascular permeability and viral spherule membrane formation during DENV-2 infection [18]. In addition, the decrease of glutathione and total antioxidant capacity in patients with dengue fever leads to mild disorder of antioxidant system [19]. 13'-Hydroxy-alpha-tocopherol regarding as an antioxidant was reported to be beneficial to thrombocytopenia due to the increase of platelets [20]. The reason for this phenomenon is associated with the antioxidant effect of vitamin E on the inflammatory response of viral infection. The increase of FFA and the abnormality of glycerin phospholipid in clinic further confirmed the important relationship between lipid metabolism and virus reproduction after dengue virus infection.

Although the differences of metabolites were identified for DF from HC, there were several limitations in our study. Firstly, all patients were infected by DENV-2 due to the outbreaks in 2017. Second, the clinic data were collected and analyzed retrospectively. Third, the sample size was small and no patients progressed to severe dengue. Therefore, the results of this study need higher quality studies for confirmation in the future.

\section{Conclusions}

In conclusion, the major clinical symptoms of DF were fever, nausea and vomiting, asthenia, skin hyperemia, leukopenia, thrombocytopenia, and increase of transaminase. In addition, DENV-2 induced alterations in human glycerophospholipids, especially for the decrease of PC, PE, SM, LPL. These results should be supported in further animal infection models and even prospective randomized controlled clinical trials.

\section{Declarations}

Ethical Approval. This study was approved by the recommendations of the Ethics Committee of The First Affiliated Hospital, College of Medicine, Zhejiang University (No. 2020622). To protect personal privacy, identifying information of each patient in the electronic database was encrypted.

Consent for publication. All authors have seen and approved the content and fulfil the journal's requirements for authorship.

Availability of data and materials. All data in the manuscript.

Competing Interests. None

Funding. This work was supported by the National Science Foundation for Young Scientists of China (No. 81803589).

Author Contributions. WY and YQQ developed the concept and designed the experiments. JPT and ZW collected clinical data from the patient records. JPT, JJZ and LZ did UPLC-Q-TOF MS spectrometer test. WY and ZW analyzed the metabonomic data. JPT and WY wrote the paper. All authors discussed the results and implications and commented on the manuscript at all stages.

Acknowledgements. Not applicable.

Authors' information. Not applicable.

\section{References}

1. Bhatt S, Gething PW, Brady OJ, Messina JP, Farlow AW, Moyes CL, Drake JM, Brownstein JS, Hoen AG, Sankoh O, Myers MF, George DB, Jaenisch T, Wint GR, Simmons CP, Scott TW, Farrar JJ, Hay SI. The global distribution and burden of dengue. Nature. 2013;496(7446):504-7. doi:10.1038/nature12060.

2. Stanaway JD, Shepard DS, Undurraga EA, Halasa YA, Coffeng LE, Brady OJ, Hay SI, Bedi N, Bensenor IM, Castañeda-Orjuela CA, Chuang TW, Gibney KB, Memish ZA, Rafay A, Ukwaja KN, Yonemoto N, Murray CJL. The global burden of dengue: an analysis from the Global Burden of Disease Study 2013. Lancet Infect Dis. 2016;16(6):712-23. doi:10.1016/S1473-3099(16)00026-8.

3. World Health Organization. Dengue and severe dengue. 2020. Available from: https://www.who.int/news-room/fact-sheets/detail/dengue-and-severedengue.

4. Shepard DS, Undurraga EA, Halasa YA, Stanaway JD. The global economic burden of dengue: a systematic analysis. Lancet Infect Dis. 2016;16(8):93541. doi:10.1016/S1473-3099(16)00146-8.

5. Liu K, Sun J, Liu X, Li R, Wang Y, Lu L, Wu H, Gao Y, Xu L, Liu Q. Spatiotemporal patterns and determinants of dengue at county level in China from 20052017. Int J Infect Dis. 2018;77:96-104. doi:10.1016/j.ijid.2018.09.003.

6. Yan H, Ding Z, Yan J, Yao W, Pan J, Yang Z, Lou X, Mao H, Lin J, Sun J, Hou J, Wu H, Wu C, Zhang Y. Epidemiological Characterization of the 2017 Dengue Outbreak in Zhejiang, China and Molecular Characterization of the Viruses. Front Cell Infect Microbiol. 2018;8:216. doi:10.3389/fcimb.2018.00216.

7. Henchal EA, Putnak JR. The dengue viruses. Clin Microbiol Rev. 1990;3(4):376-96. doi:10.1128/cmr.3.4.376.

8. Raafat N, Blacksell SD, Maude RJ. A review of dengue diagnostics and implications for surveillance and control. Trans R Soc Trop Med Hyg. 2019;113(11):653-60. doi:10.1093/trstmh/trz068.

9. Chang D, Weljie A, Newton J. Leveraging latent information in NMR spectra for robust predictive models. Pac Symp Biocomput. 2007:115-26.

10. Wilder-Smith A, Ooi EE, Horstick O, Wills B. Dengue Lancet. 2019;393(10169):350-63. doi:10.1016/S0140-6736(18)32560-1.

11. Yan H, Ding Z, Yan J, Yao W, Pan J, Yang Z, Lou X, Mao H, Lin J, Sun J, Hou J, Wu H, Wu C, Zhang Y. Epidemiological Characterization of the 2017 Dengue Outbreak in Zhejiang, China and Molecular Characterization of the Viruses. Front Cell Infect Microbiol. 2018 Jul 4;8:216. doi: 10.3389/fcimb.2018.00216.

12. Potts JA, Thomas SJ, Srikiatkhachorn A, Supradish PO, Li W, Nisalak A, Nimmannitya S, Endy TP, Libraty DH, Gibbons RV, Green S, Rothman AL, Kalayanarooj S. Classification of dengue illness based on readily available laboratory data. Am J Trop Med Hyg. 2010;83(4):781-8.

doi:10.4269/ajtmh.2010.10-0135. 
13. Trung DT, Thao le TT, Hien TT, Hung NT, Vinh NN, Hien PT, Chinh NT, Simmons C, Wills B. Liver involvement associated with dengue infection in adults in Vietnam. Am J Trop Med Hyg. 2010;83(4):774-80. doi:10.4269/ajtmh.2010.10-0090.

14. Cui L, Lee YH, Kumar Y, Xu F, Lu K, Ooi EE, Tannenbaum SR, Ong CN. Serum metabolome and lipidome changes in adult patients with primary dengue infection. PLoS Negl Trop Dis. 2013;7(8):e2373. doi:10.1371/journal.pntd.0002373.

15. Perera R, Riley C, Isaac G, Hopf-Jannasch AS, Moore RJ, Weitz KW, Pasa-Tolic L, Metz TO, Adamec J, Kuhn RJ. Dengue virus infection perturbs lipid homeostasis in infected mosquito cells. PLoS Pathog. 2012;8(3):e1002584. doi:10.1371/journal.ppat.1002584.

16. Heaton NS, Randall G. Dengue virus-induced autophagy regulates lipid metabolism. Cell Host Microbe. 2010;8(5):422-32. doi:10.1016/j.chom.2010.10.006.

17. Voge NV, Perera R, Mahapatra S, Gresh L, Balmaseda A, Loroño-Pino MA, Hopf-Jannasch AS, Belisle JT, Harris E, Blair CD, Beaty BJ. Metabolomics-Based Discovery of Small Molecule Biomarkers in Serum Associated with Dengue Virus Infections and Disease Outcomes. PLoS Negl Trop Dis. 2016;10(2):e0004449. doi:10.1371/journal.pntd.0004449.

18. Byers NM, Fleshman AC, Perera R, Molins CR. Metabolomic Insights into Human Arboviral Infections: Dengue, Chikungunya, and Zika Viruses. Viruses. 2019,11(3). pii: E225. doi: 10.3390/v11030225.

19. Klassen P, Biesalski HK, Mazariegos M, Solomons NW, Fürst P. Classic dengue fever affects levels of circulating antioxidants. Nutrition. $2004 ; 20(6): 542-7$. doi:10.1016/j.nut.2004.03.016.

20. Vaish A, Verma S, Agarwal A, Gupta L, Gutch M. Effect of vitamin E on thrombocytopenia in dengue fever. Ann Trop Med Public Health. 2012;5:282-5. doi:10.4103/1755-6783.102004.

\section{Tables}


Table 1

Clinical characteristics of DF

\begin{tabular}{|c|c|c|c|}
\hline \multicolumn{2}{|l|}{ General Information } & \multicolumn{2}{|l|}{ Laboratory examinations } \\
\hline Age (Year) & $53.9 \pm 16.3$ & WBC (3.5-9.5)*10E9/L & $2.7 \pm 1.5$ \\
\hline Gender (Males/Females) & $21 / 29$ & $L(20-50) \%$ & $31.2 \pm 14.7$ \\
\hline $\mathrm{BMI}(\mathrm{kg} / \mathrm{m} 2)$ & $22.6 \pm 3.0$ & $M(3-10) \%$ & $10.5 \pm 4.3$ \\
\hline Temperature $\left({ }^{\circ} \mathrm{C}\right)$ & $39.1 \pm 0.8$ & $N(40-75) \%$ & $57.0 \pm 15.3$ \\
\hline Admission days & $4.9(3.9-7)$ & $\mathrm{Hb}(115-150) \mathrm{g} / \mathrm{L}$ & $138.1 \pm 17.0$ \\
\hline Clinical symptoms & & PLT $(125-350) * 10 E 9 / L$ & $65.9 \pm 40.8$ \\
\hline Rash & $36 \%(18 / 50)$ & $\mathrm{CRP}(0-10) \mathrm{mg} / \mathrm{L}$ & $8.7 \pm 14.2$ \\
\hline Petechia & $38 \%(19 / 50)$ & Alb $(40-55) \mathrm{g} / \mathrm{l}$ & $36.2 \pm 2.8$ \\
\hline Nausea and vomiting & $68 \%(34 / 50)$ & $\operatorname{ALT}(7-40) \mathrm{U} / \mathrm{L}$ & $64.4 \pm 53.0$ \\
\hline Abdominal pain and diarrhea & $64 \%(32 / 50)$ & AST (13-35) U/L & $114.1 \pm 83.7$ \\
\hline Asthenia & $98 \%(49 / 50)$ & GGT (7-45) U/L & $91.2 \pm 127.8$ \\
\hline Myalgia & $60 \%(30 / 50)$ & Tbil (3.4-24) $\mu \mathrm{mol} / \mathrm{L}$ & $10.9 \pm 5.3$ \\
\hline Headache & $46 \%(23 / 50)$ & $\mathrm{Cr}(35-123) \mu \mathrm{mol} / \mathrm{L}$ & $69.7 \pm 12.4$ \\
\hline Skin hyperemia & $50 \%(25 / 50)$ & $\mathrm{BuN}(3.1-8.8) \mathrm{mmol} / \mathrm{L}$ & $3.8 \pm 1.5$ \\
\hline Lymphadenectasis & $8 \%(4 / 50)$ & FFA (129-769) $\mu \mathrm{mol} / \mathrm{L}$ & $923.6 \pm 353.0$ \\
\hline Cough and sputum production & $36 \%(18 / 50)$ & $\mathrm{LDH}(120-250) \mathrm{U} / \mathrm{L}$ & $358.9 \pm 112.1$ \\
\hline Dyspnea & $10 \%(5 / 50)$ & CK $(40-200) \mathrm{U} / \mathrm{L}$ & $298.1 \pm 337.4$ \\
\hline Haematemesis & $4 \%(2 / 50)$ & $\mathrm{TC}(3.11-5.96) \mathrm{mmol} / \mathrm{L}$ & $3.3 \pm 0.8$ \\
\hline Hemorrhinia and gingival bleeding & $6 \%(3 / 50)$ & $\mathrm{TG}(0.34-1.7) \mathrm{mmol} / \mathrm{L}$ & $1.6 \pm 0.6$ \\
\hline Comorbidities & & PT $(9.7-13.5) \mathrm{s}$ & $11.1 \pm 1.1$ \\
\hline Hypertension & $26 \%(13 / 50)$ & $\mathrm{Fi}(2-4) \mathrm{g} / \mathrm{L}$ & $2.1 \pm 0.4$ \\
\hline Diabetes mellitus & $10 \%(5 / 50)$ & РСТ $(0-0.25) \mathrm{ng} / \mathrm{ml}$ & $0.2 \pm 0.2$ \\
\hline Fatty liver & $14 \%(7 / 50)$ & Treatment & \\
\hline Hypothyroidism & $4 \%(2 / 50)$ & Platelet transfusion & $18 \%(9 / 50)$ \\
\hline Coronary heart disease & $2 \%(1 / 50)$ & Hemagglutinase & $32 \%(16 / 50)$ \\
\hline Cancer & $4 \%(2 / 50)$ & Human granulocyte-stimulating Factor & $38 \%(19 / 50)$ \\
\hline Ankylosing spondylitis & $2 \%(1 / 50)$ & Recombinant human interleukin-11 & $30 \%(15 / 50)$ \\
\hline Bronchiectasis & $2 \%(1 / 50)$ & Tranexamic acid & $18 \%(9 / 50)$ \\
\hline Chronic hepatitis B & $2 \%(1 / 50)$ & Fibrinogen & $8 \%(4 / 50)$ \\
\hline
\end{tabular}


Table 2

17 metabolites that show differences between DF and HC

\begin{tabular}{|c|c|c|c|c|c|c|c|c|}
\hline Compound & $\begin{array}{l}\text { RT } \\
(\min )\end{array}$ & VIP & $\mathbf{p}$ & Adducts & Description & $\mathrm{m} / \mathrm{z}$ & Formula & $\begin{array}{l}\text { Fragment } \\
\text { Score }\end{array}$ \\
\hline 17.20_782.5694n & 7.86 & 22.23 & * & $\begin{array}{l}\mathrm{M}+\mathrm{H} \\
\mathrm{M}+\mathrm{Na}\end{array}$ & LysoPC(18:2(9Z,12Z)) & 520.3417 & C26H50NO7P & 75.8 \\
\hline $16.25 \_782.5721 \mathrm{~m} / \mathrm{z}$ & 16.25 & 19.06 & * & $\mathrm{M}+\mathrm{H}$ & PC(18:0/18:4(6Z,9Z,12Z,15Z)) & 782.5721 & C44H80N08P & 6.15 \\
\hline 17.20_807.5815n & 17.20 & 18.89 & * & $\begin{array}{l}\mathrm{M}+\mathrm{H} \\
\mathrm{M}+\mathrm{Na}\end{array}$ & $\operatorname{PE}(21: 0 / 20: 5(5 Z, 8 Z, 11 Z, 14 Z, 17 Z))$ & 808.5888 & $\mathrm{C} 46 \mathrm{H} 82 \mathrm{NO} 8 \mathrm{P}$ & 17.4 \\
\hline 15.96_779.5492n & 15.96 & 11.83 & * & $\begin{array}{l}\mathrm{M}+\mathrm{H} \\
\mathrm{M}+\mathrm{Na}\end{array}$ & PC(20:5(5Z,8Z,11Z,14Z,17Z)/16:0) & 780.5565 & C44H78N08P & 64.5 \\
\hline 20.19_785.5958n & 20.19 & 10.36 & * & $\begin{array}{l}M+H, \\
M+K, \\
M+H- \\
H 2 O\end{array}$ & PC(18:1(9Z)/18:1(9Z)) & 786.6031 & C44H84N08P & 42.4 \\
\hline 20.19_771.6136n & 20.19 & 9.59 & * & $\begin{array}{l}M+N a \\
M+K \\
M+H\end{array}$ & PC(18:0/P-18:1(11Z)) & 810.6036 & C44H86N07P & 5.04 \\
\hline 17.76_783.5809n & 17.76 & 8.80 & 0.001 & $\begin{array}{l}\mathrm{M}+\mathrm{H}- \\
\mathrm{H} 2 \mathrm{O}, \mathrm{M} \\
+\mathrm{H}\end{array}$ & $\mathrm{PC}(18: 1(11 Z) / 18: 2(9 Z, 12 Z))$ & 784.5863 & $\mathrm{C} 44 \mathrm{H} 82 \mathrm{NO} 8 \mathrm{P}$ & 1.44 \\
\hline 16.31_756.5570m/z & 16.31 & 7.51 & * & $\mathrm{M}+\mathrm{H}$ & $\mathrm{PC}(16: 0 / 18: 3(6 Z, 9 Z, 12 Z))$ & 756.557 & $\mathrm{C} 42 \mathrm{H} 78 \mathrm{~N} 08 \mathrm{P}$ & 0.412 \\
\hline 15.95_756.5567m/z & 15.95 & 7.11 & * & $\mathrm{M}+\mathrm{H}$ & $\mathrm{PC}(14: 0 / 20: 3(5 Z, 8 Z, 11 Z))$ & 756.5567 & C42H78N08P & 5.61 \\
\hline 17.66_847.5400n & 17.66 & 6.75 & 0.001 & $\begin{array}{l}\mathrm{M}+\mathrm{H} \\
\mathrm{M}+ \\
\mathrm{NH} 4\end{array}$ & PS(19:1(9Z)/22:6(4Z,7Z,10Z,13Z,16Z,19Z)) & 865.5344 & C47H78N010P & 0.244 \\
\hline 7.85_543.3333n & 7.85 & 6.39 & * & $\begin{array}{l}\mathrm{M}+\mathrm{H} \\
\mathrm{M}+\mathrm{Na}\end{array}$ & LysoPC(20:4(8Z,11Z,14Z,17Z)) & 544.3406 & C28H50NO7P & 41.6 \\
\hline 15.81_729.5335n & 15.81 & 4.87 & 0.001 & $\begin{array}{l}\mathrm{M}+\mathrm{H} \\
\mathrm{M}+\mathrm{Na}\end{array}$ & $\mathrm{PC}(14: 1(9 Z) / 18: 1(11 Z))$ & 752.5357 & $\mathrm{C} 40 \mathrm{H} 76 \mathrm{NO} 8 \mathrm{P}$ & 95.8 \\
\hline 18.01_446.3751n & 18.01 & 4.18 & * & $\begin{array}{l}\mathrm{M}+\mathrm{H}- \\
\mathrm{H} 2 \mathrm{O}, \mathrm{M} \\
+\mathrm{H}\end{array}$ & 13'-Hydroxy-alpha-tocopherol & 429.3718 & $\mathrm{C} 29 \mathrm{H} 5003$ & 70.9 \\
\hline $16.76 \_744.5570 \mathrm{~m} / \mathrm{z}$ & 16.76 & 3.42 & * & $M+K$ & $\operatorname{SM}(\mathrm{d} 18: 0 / 16: 0)$ & 744.557 & $\mathrm{C} 39 \mathrm{H} 82 \mathrm{~N} 2 \mathrm{O} 6 \mathrm{P}+$ & 11.3 \\
\hline 9.88_531.2744m/z & 9.88 & 1.71 & 0.018 & $\mathrm{M}+\mathrm{H}$ & PG(20:5(5Z,8Z,11Z,14Z,17Z)/0:0) & 531.2744 & $\mathrm{C} 26 \mathrm{H} 4309 \mathrm{P}$ & 0 \\
\hline 7.01_444.2715n & 7.01 & 1.31 & 0.001 & $\begin{array}{l}\mathrm{M}+\mathrm{H} \\
\mathrm{M}+\mathrm{Na} \\
\mathrm{M}+ \\
\mathrm{NH} 4, \mathrm{M} \\
+\mathrm{H}-\mathrm{H} 2 \mathrm{O}\end{array}$ & 1(3)-glyceryl-6-keto-PGF1alpha & 445.2787 & $\mathrm{C} 23 \mathrm{H} 4008$ & 20.6 \\
\hline 8.65_730.4595n & 8.65 & 1.28 & 0.004 & $\begin{array}{l}\mathrm{M}+ \\
\mathrm{NH} 4, \mathrm{M} \\
+\mathrm{Na}, \mathrm{M} \\
+\mathrm{H}-\mathrm{H} 2 \mathrm{O}\end{array}$ & $\mathrm{PA}(17: 2(9 Z, 12 Z) / 22: 6(4 Z, 7 Z, 10 Z, 13 Z, 16 Z, 19 Z))$ & 748.4933 & $\mathrm{C} 42 \mathrm{H} 6708 \mathrm{P}$ & 0 \\
\hline
\end{tabular}

RT, retention time; VIP, value of variable importance in the projection; * $p<0.001$.

\section{Figures}




\section{HMDB}

Homogeneous non-metal compounds

Organic 1,3-dipolar compounds

Organic Polymers

Organophosphorus compounds

Organic compounds

Organohalogen compounds

Organonitrogen compounds

Lignans, neolignans and related compounds

Hydrocarbons

Organic nitrogen compounds

Organosulfur compounds

Organooxygen compounds

Alkaloids and derivatives

Nucleosides, nucleotides, and analogues

Benzenoids

Organic oxygen compounds

Phenylpropanoids and polyketides

Organic acids and derivatives

Organoheterocyclic compounds

Lipids and lipid-like molecules

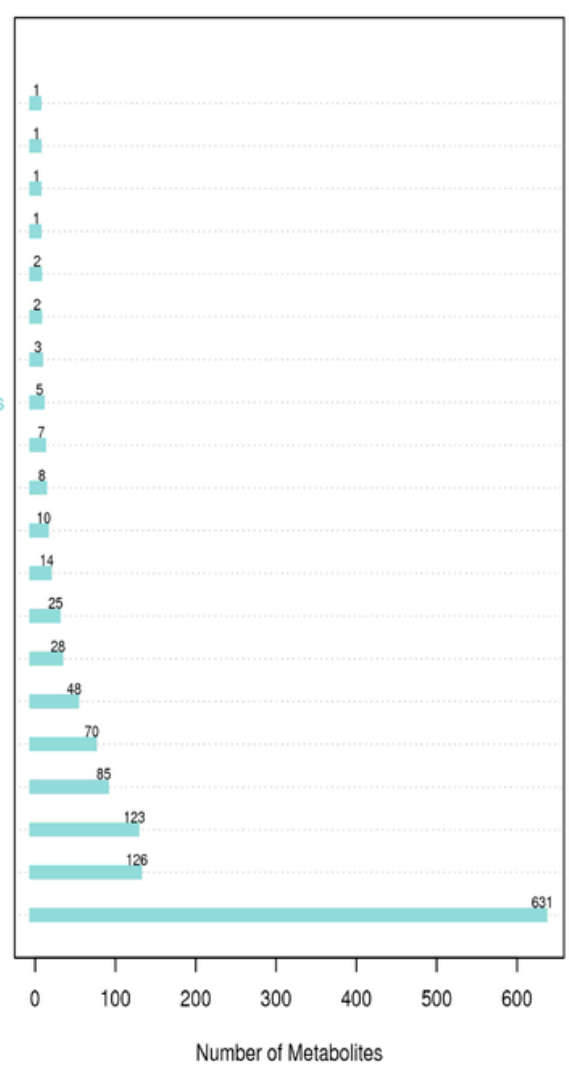

Fatty Acyls [FA]

Elcosanoids [FAOS]

Glycerolipids [GL]

Lonoradylycerols [G[01]

Eilycosylmonoradyiglycerols [GLO4]

Glycerophospholipids [GP]

Oither Glycerophospholitids [GP OOP

Glycosylalycerophospholipids [GP -4]

Glyceropyrophosphates [CP11]

Gycerophosphoserines GP

Glycerophosphoinositols [GPO6

Glycerophosphoglycerols [GP04]

Glycerophosphoethanolamines [GP02]

Glycerophosphocholines [GP01]

Givcerophosphates [GP10]

Polyketides [PK]

lon-ribosomal peptide/polyketide hybrids [PK14] Tacrolides and actone polyketides [PKO4]

Havonoids [PK12]

Ansamycins and related polyketides [PK05]

\section{Prenol Lipids [PR]}

Prontinones and

Polyprenols [PR03]

Hoprenoids [PRoul]

Saccharolipids [SL]

ther acyl sugars [SL05]

Sterol Lipids [ST]

steroids [ST02]

Steroid conjugates [ST05]

Secosteroids [ST03]
Bile acids and derivatives [STO4]

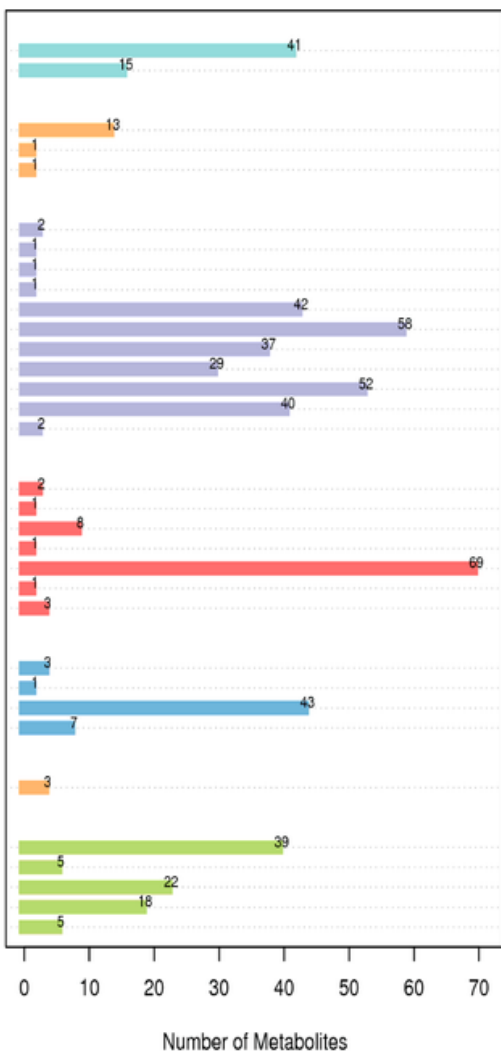

Number of Metabolites

Figure 1

Metabolites annotation. (a) HMDB annotation; (b) LIPID MAPS annotation. 

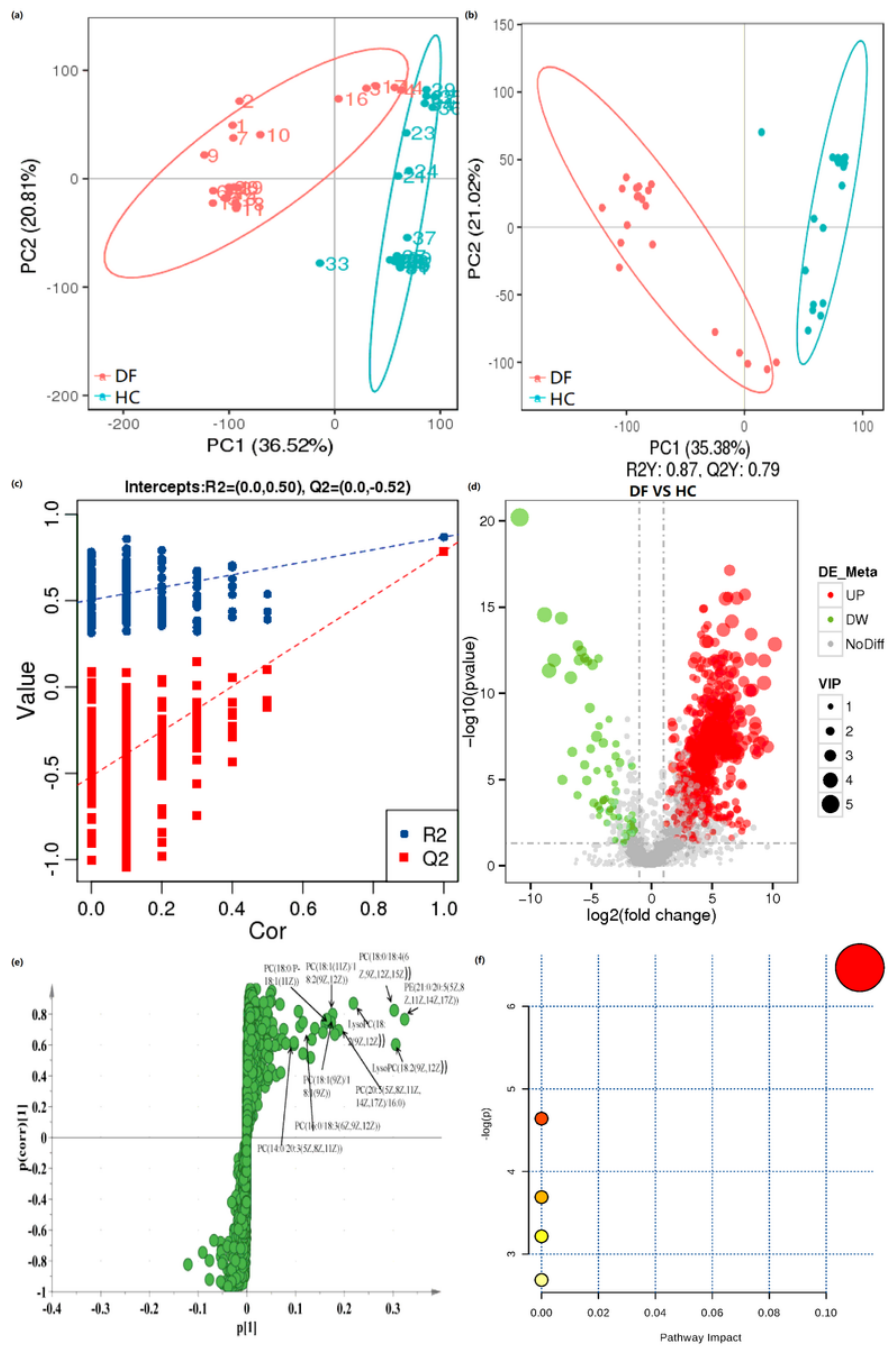

\section{Figure 2}

Multivariate analysis of DF and HC. (a) PCA; (b) PLS-DA score plots; (c) Model validation; (d)Volcanic map of differential metabolites; (e) OPLS-DA S-plot of metabolites; (f) Pathway analysis.

\section{Supplementary Files}

This is a list of supplementary files associated with this preprint. Click to download.

- SupplementaryFigure1.png 